Dipetrives

\title{
Proposta de metodologia de busca de evidências em Atenção Primária á Saúde: o exemplo da osteoporose em homens
}

\author{
A proposal of a methodology for evidence search in \\ primary health care: the example of osteoporosis in men
}

Leonardo Cançado Monteiro Savassi ${ }^{1}$ Ana Carolina Diniz Oliveira ${ }^{2}$ Ruth Borges Dias ${ }^{3}$

\section{Resumo}

O Médico de Família e Comunidade deve buscar a melhor evidência disponível, mas a dificuldade de acesso à informação científica ou a centros acadêmicos em Atenção Primária à Saúde é uma realidade global. A busca de informações sistematizadas não demanda tecnologias extensas ou a necessidade do médico de ambulatório ser um cientista ou pesquisador. Por meio de uma sistematização didática, podem-se recuperar artigos que apresentem boa evidência clínica. O objetivo deste artigo é apresentar uma forma simplificada e sistematizada de busca de evidências científicas para a Atenção Primária à Saúde em cinco passos. Por meio do exemplo de uma dúvida da prática clínica referente ao diagnóstico da osteoporose em homens, realizou-se busca sistematizada em bases de dados, encontrando diagnóstico através da densitometria (DEXA) como única opção baseada em evidências, o que dificulta seu manejo no sistema público de saúde, embora alguns guidelines defendam o diagnóstico em casos de fratura de fragilidade. Discutem-se as evidências encontradas sob a ótica da validação externa com individualização das evidências para o paciente.

\begin{abstract}
The Family Physician should always seek the best available evidence, however the difficult access to scientific information or academic centers in primary care is a worldwide reality. The search for systematized information does not demand extensive technologies nor must the doctor be a scientist or researcher. Didactic systematization allows recovering articles presenting good clinical evidence. The purpose of this article is to present a simplified and systematized search method for scientific evidence in the field of primary care in five steps using the example of a question in the clinical practice regarding the diagnosis of osteoporosis affecting men. A systematized search in databases resulted in densitometry (DEXA) as the only evidence-based possibility of diagnosis, hampering its management in the public health system although some guidelines define OSTEOPOROSIS when fragility fractures are present. The evidences found are discussed from the perspective of external validation, individualizing the evidences for patients.
\end{abstract}

Palavras-chave: Medicina Baseada em

Evidências, Bases de Dados Bibliográficas,

Atenção Primária à Saúde, Osteoporose.
Key Words: Evidence-based medicine; Databases Bibliographic; Primary Health Care; Osteoporosis.

${ }^{1}$ Coordenador da Residência Médica em Medicina de Família e Comunidade, Hospital Público Regional de Betim-MG, Betim, Minas Gerais, Brasil. ${ }_{3}^{2}$ Médica, Prefeitura Municipal de Belo Horizonte, Belo Horizonte, Minas Gerais, Brasil.

${ }^{3}$ Presidente da AMMFC Gestão 2007-2009, Docente, Universidade de Alfenas Campus BH, Belo Horizonte, Minas Gerais, Brasil. 


\section{Introdução}

O avanço tecnológico torna cada vez mais difícil manter-se atualizado. Alguns fatos dão a verdadeira dimensão da produção científica. A base Medline congrega títulos e resumos de artigos de cerca de 33.069 revistas mundiais, algumas delas brasileiras ${ }^{1}$. Por ano são acrescentados cerca de 500.000 novos artigos, o que representa cerca de 9.600 por semana e 1.400 por $\mathrm{dia}^{2}$.

Dois exemplos clássicos ilustram a importância da busca da informação atualizada e da melhor evidência científica: Bernardo ${ }^{3}$ relata que, a despeito de uma tecnologia da informação inexistente à época, a não preocupação com a evidência científica protelou em cerca de 15 anos o uso de trombolíticos para Infarto Agudo do Miocárdio (IAM). Os trabalhos publicados nos anos 1960 e 1970 já demonstravam o benefício dessas drogas no tratamento do IAM, porém somente 15 anos depois os livros de especialidades passaram a recomendar seu uso rotineiro às custas de muitos óbitos evitáveis.

Outro exemplo é o do tratamento da Infecção do Trato Urinário em crianças ${ }^{4}$ Os regimes de curta duração dose única a três dias - são consagrados em adultos, e vários Estudos Randomizados e Controlados não demonstraram diferença estatística em relação a tratamento de longa duração - 7 a 10 dias - na criança. Foi necessário realizar uma revisão sistemática, e posterior meta-análise, que, após selecionar artigos válidos, demonstrou menos falhas de tratamento na terapia de longa duração. Tal conclusão só foi possível porque, ao se revisar vários estudos, a amostra de pacientes estudada foi grande o suficiente para demonstrar diferença significativa entre os tratamentos.

Uma revisão narrativa ou clínica é diferente de uma revisão sistemática. Esta é elaborada com rigor metodológico e constitui-se na melhor evidência científica disponível' Artigos de revisão são documentos de atualização do conhecimento sobre um tema, mediante a revisão de literatura disponível, tendo maior difusão e utilização entre os profissionais da $\mathrm{APS}^{3}$. Quando diversos textos de natureza opinativa são articulados por um conjunto de autores, habitualmente de uma mesma especialidade ou sociedades afins, compõe-se um Consenso, que pode atender a interesses de natureza corporativa.

A utilidade (U) de uma informação a se recuperar está ligada a três conceitos-chave: a relevância $(\mathrm{R})$ da informação encontrada, a validade (V) desta informação para a prática, e o trabalho (W) necessário para acessá-la

$$
U=\frac{V \times R}{W}
$$

A validade de um artigo guarda relação ainda com a sua aplicabilidade. A validade interna refere-se à estrutura própria do estudo, se os resultados e suas interpretações são coerentes com a metodologia utilizada, e se esta foi adequada para responder a(s) pergunta(s) formulada(s) ${ }^{3}$. Validade externa refere-se às conclusões do estudo, se são generalizáveis a outras populações e se podem ser aplicadas na prática clínica diária do profissional ${ }^{6}$.

Uma revisão clínica geralmente inicia-se a partir de uma dúvida da vida prática. Formula-se, então, uma pergunta estruturada, identificando palavras-chave ou descritores que serão a base da busca nos bancos de dados científicos disponíveis ${ }^{3}$. Entretanto, para o médico pouco habilitado à busca de informação científica em bancos de dados, nem sempre é fácil fazer a análise crítica da melhor evidência, e ela pode não estar disponível para a vários problemas da prática clínica.

A melhor evidência clínica para responder a um problema idealmente é resultante de um ensaio clínico randomizado ou de uma revisão sistemática de ensaios, sendo a menos confiável aquela proveniente de Fóruns de Especialistas (Quadro 1) ${ }^{7}$. Guidelines que explicitam o nível e as fontes de evidência e graus de recomendação geralmente constituem-se em boas fontes para a tomada de decisão, já que, de modo ideal, deve-se iniciar a leitura por fontes mais elaboradas, prontas para responder questões objetivas, que não demandem formação em leitura crítica e ocupem menos tempo ${ }^{3}$.

Uma metodologia sistematizada de busca de informações é apresentada por Cárceres e Gonzalez ${ }^{8}$, com passos 
Quadro 1. Níveis de evidência

\section{Níveis de Evidência Científica nos Enforques de Terapia, Prevenção e Etiologia/Risco}

Nível I: ensaio clínico randomizado (ECR) ou revisão sistemática (RS) de ECRs com desfechos clínicos (ser duplo-cego, ter alocação sigilosa, completar acompanhamento de $>80 \%$ e explicitar poder adequado $(<0,80, \alpha=0,05)$;

Nível II: ECR ou RS de ECR de menor qualidade:

- com desfechos substitutos valiados (desfechos substitutos são considerados valiados quando há demostração, em ECR de longa duração, de que mudanças entre os desfechos substitutos e clínicos são correlacionadas e de que o substituto capta plenamente todos os efeitos da terapia);

- com análise de subgrupos ou de hipóteses a posteriori;

- com desfechos clínicos, mas de menor rigor metodológico;

Estudo observacional de reconhecimento peso científico (coorte ou caso-controle aninhado em uma coorte, séries temporais múltiplas) ou revisão sistemática desses estudos;

Nível III: ECR com desfechos substitutos não-avaliados; Estudo de Caso-controle;

Nível IV: Estudo com desfecho clínico, mas com maior potencial de viés (tal como experimento não comparado e demais estudos observacionais);

Nível V: Fórum representativo ou opinião de especialista sem evidências dos níveis supracitados.

Fonte: Duncan, 20047.

detalhados de busca de palavras-chave, posteriormente de guidelines, revisões sistemáticas e finalmente de artigos originais. Com base neste esquema, o que se propõe aqui é adequá-la a uma realidade brasileira, excluindo aquelas fontes que não são de livre acesso (Open Access) e incluindo fontes nacionais e internacionais relevantes para a Atenção Primária à Saúde.

\section{Metodologia, Materiais e Métodos}

Para conseguir resultados adequados deve-se utilizar uma metodologia confiável de busca que permita recuperar informações de qualidade em um tempo curto e em quantidade viável. Há uma sequência clássica de etapas a serem seguidas para obter a decisão clínica adequada":

I. Formulação adequada da pergunta (passos 1 e 2).

II. Busca da evidência (passos 3 a 5).

III. Análise qualitativa da evidência obtida.

IV. Análise quantitativa da evidência obtida.

V. Análise de custo-efetividade.

VI. Avaliação da aplicabilidade dos dados para a sua prática clínica.
Como iniciar a pesquisa bibliográfica:

Passo 1. Uma forma de encontrar a resposta apropriada a uma dúvida surgida no atendimento do paciente é formular a pergunta por meio do acrônimo P.I.C.O. ${ }^{10}$, no qual:

$\mathrm{P}=$ paciente ou população

$\mathrm{I}=$ intervenção ou indicador

$\mathrm{C}=$ comparação ou controle

$\mathrm{O}=$ Outcome ou desfecho

Da mesma forma, o acrônimo P.P.R. representa os elementos de pesquisa a serem abordados, de maneira simplificada ${ }^{11}$ :

$\mathrm{P}=$ Problema

$\mathrm{P}=$ Preditor (fator de risco/prognóstico/intervenção)

$\mathrm{R}=$ Resultado

Passo 2. Determinar as palavras-chave, utilizando o MeSH Database do Pubmed ou o banco de dados DeCS (Descritores em Ciência da Saúde) da Biblioteca Virtual em Saúde. Tal passo é importante para que se busque o unitermo correto e se recupere o maior número de artigos que tenham relação com o tema. 
Passo 3. Uma vez localizada a palavra-chave, há a opção de busca por guidelines, sempre com uma leitura crítica sobre os vieses de interesses. Para isso, deve-se avaliar o âmbito no qual eles foram desenvolvidos e quais são os interesses dessas associações quanto ao guideline em questão (apropriação coorporativa de pacientes ou favorecimento de tecnologias próprias destas associações). Outro ponto fundamental é avaliar se os guidelines foram desenvolvidos no âmbito da Atenção Primária à Saúde ou no nível Comunitário, ou se o ambiente de desenvolvimento do guideline é hospitalar ou da atenção especializada. Sugerem-se os seguintes repositórios de guidelines.

NICE - National Institute for Health and Clinical Excellence - http://www.nice.org.uk.

National Eletronic Library for Health - http:/ / rms.nelh.nhs .uk/guidelinesfinder.

CMA Infobase - http://mdm.ca/cpgsnew/cpgs/index.asp. Diretrizes Médicas, da Associação Médica Brasileira e Conselho Federal de Medicina - http://www.projeto diretrizes.org.br.

National Guideline Clearinghouse - http:/ / www.guideline .gov. USPSTF - US Preventive Services Task Force (recupera artigos relacionados a prevenção clínica) - http:/ /www.ahrq .gov/clinic/uspstfix.htm.

CTFPHC - Canadian Task Force on Preventive Health Care - http://www.ctfphc.org/

Sítios oficiais de sociedades de especialidade nacionais e internacionais, que trabalhem com o tema em questão.

Passo 4. Busca de revisões sistemáticas. Uma forma simples de localização consiste em utilizar a opção Clinical Queries com filtros metodológicos pré-estabelecidos, acessível na página principal de Pubmed/Medline (no menu localizado à esquerda da página) ou diretamente no endereço eletrônico infracitado, clicando na opção "Find Systematic Reviews". A Colaboração Cochrane se apresenta como a principal fonte de revisões sistemáticas em saúde. Novamente, cabe ressaltar o cuidado de avaliar o ambiente em que os artigos selecionados para a revisão se desenvolvem, tendo em vista que achados de centros subespecializados ou de âmbito hospitalar podem não ser aplicáveis para a
APS.

- PubMed Clinical Queries - http://www.ncbi.nlm.nih.gov/ entrez/query/static/clinical.html.

- Cochrane/Bireme-http://cochrane.bireme.br/cochrane/ main.

Passo 5. Para certificar-se de que a revisão realizada encontrase atualizada, deve ser completada a busca, utilizando artigos originais recentes. Neste âmbito, é interessante ordenar os artigos por data, selecionar aqueles publicados nos anos seguintes às Revisões Sistemáticas ou guidelines encontrados, ou ao menos nos últimos dois anos:

- PubMed - http://www.ncbi.nlm.nih.gov/sites/entrez.

- Bireme/BVS - http://www.bireme.br; a busca no site da bireme/BVS inclui a busca na base de dados da LILACS (Literatura Latino Americana e do Caribe em Ciências da Saúde) e do SciELO (Scientific Literature Online).

- DARE - http://www.york.ac.uk/inst/crd/.

- American College of Physicians (ACP) Journal Club http://www.acponline.org/journals/ acpjc/jcmenu.htm.

A partir desse ponto, com a bibliografia levantada, passa-se para a análise qualitativa (etapa III) e quantitativa (etapa IV) da evidência obtida, do custo-efetividade das medidas adotadas (etapa V), da aplicabilidade para a prática clínica (etapa VI), ou seja, validades interna e externa dos artigos.

\section{Resultados}

Uma dúvida da prática clínica:

Paciente, de 67 anos, sedentário, portador de sequelas neuromotoras de hanseníase, que dificultavam parcialmente a deambulação, sem outras comorbidades ou alterações de equilíbrio, apresentou fratura de fêmur após queda da própria altura e, desde então, passou a depender de andador com piora da qualidade de vida e da autonomia e redução dos escores de Atividades de Vida Diárias.

O que diz a Literatura Clássica:

Livros textos que abordam Osteoporose a definem e classificam pela Densitometria Óssea, a partir do achado de densidade mineral óssea (DMO) abaixo de 2,5 desvios-padrão da referência, de acordo com a Organiza- 
ção Mundial de Saúde (OMS). O quadro é classificado em tipo I ou "pós-menopausa" e osteoporose do tipo II ou "senil", esta a que afeta homens ${ }^{12}$.

A perda óssea senil em homens pode ser secundária a hipogonadismo, corticoterapia, ressecção gástrica, hipercalciúria idiopática e abuso de álcool. Até $25 \%$ deles com fratura de quadril ou coluna apresentam deficiência androgênica ${ }^{12}$. Em geral, fraturas de fêmur proximais (FFP) ocorrem antes dos 80 anos, especialmente em homens, responsáveis por $1 / 3$ de todas elas com morbidade e mortalidade piores. A taxa de mortalidade para mulheres com FFP é de quase $20 \%$ nos primeiros três meses, e, nos homens, o dobro com dependência para atividades de vida em $50 \%$ deles ${ }^{13}$.

Biomarcadores ósseos se restringem à monitorização terapêutica. A radiografia convencional tem precisão e sensibilidade fracas e, sem fratura vertebral, não se diagnostica osteoporose. As alterações radiológicas seriam visíveis somente com perdas da densidade acima de 30\%, representando diagnóstico tardio, inviabilizando a prevenção $^{12,13}$. O ultrassom ósseo não tem correlação com a densitometria, com parâmetros não estabelecidos para medir massa óssea, além de limitações, artefatos e problemas técnicos. A principal fonte nacional em $\mathrm{APS}^{7}$ se refere exclusivamente a mulheres.

Observando que a literatura tradicional não responde plenamente a questão, e que novas evidências podem ter surgido após a confecção dos livros textos, procurouse na literatura novas evidências para o diagnóstico e a indicação de tratamento da osteoporose em homens. Sugerese que o leitor siga os passos identificados neste estudo para maior didática do aprendizado.

A busca da informação:

A grande dúvida levantada é quando pedir a Densitometria e/ou suspeitar de Osteoporose e se todo homem acima de 65 anos deveria ser triado, o que inclui avaliar fatores de risco. Avaliou-se também opções à densitometria óssea, exame de difícil acesso na rede pública, possibilidade de firmar diagnóstico por achados clínicos ou outros exames, e a possibilidade de tratar o paciente em questão a despeito da realização da Densitometria. Para isso realizou-se pesquisa em Novembro de 2007 nas prinprincipais bases científicas, seguindo os passos propostos:

Passo 1. Formulação da pergunta:

Em homens, quais seriam os métodos diagnósticos disponíveis para a Osteoporose (Senil/ relacionada à idade)?

Utilizou-se acrômio PICO, para definir a pergunta estruturada:

P: Homens.

I/C: Métodos Diagnósticos.

O: Diagnóstico de Osteoporose.

Passo 2. O DeCS ${ }^{14}$ traduz "Osteoporose" como "Osteoporosis". No Mesh database ${ }^{15}$ o unitermo "Osteoporosis" recupera "osteoporosis" e "osteoporosis postmenopausal". A definição de "Osteoporosis" no Mesh database é: "reduction of bone mass without alteration in the composition of bone, leading to fractures. Primary osteoporosis can be of two major types: postmenopausal osteoporosis and age-related or senile osteoporosis"

Foram então definidos os unitermos "Osteoporosis", "Diagnosis" e "men" para a pesquisa em bancos de dados científicos.

Passo 3. Uma vez localizada a palavra-chave, realizou-se busca de guidelines nos sítios de referência supracitados: NELH, NICE, CMA Infobase, Projeto Diretrizes e NGC. Além disso, no USPSTF e CTFPHC. A busca foi complementada nestes sítios oficiais: Sociedade Brasileira de Medicina de Família e Comunidade (www.sbmfc .org.br), Clínica Médica (www.sbcm.org.br), Geriatria e Gerontologia (www.sbgg.org.br) e Sociedade Brasileira de Osteoporose (http://www.sobrao.com.br). Ela foi realizada também na National Osteoporosis Foundation (www.nof.org) e na Osteoporosis Society of Canada (www.osteoporosis .ca), a partir de citações dentro dos guidelines internacionais. O resultado da busca por guidelines está explicitado no Quadro 2. 
Quadro 2. Locais de busca e evidência encontrada.

\begin{tabular}{|ll}
\hline Fontes de Guidelines & \multicolumn{1}{c}{ Informação Encontrada } \\
\hline NELH (NHS): & $\begin{array}{l}\text { Canadian Consensus Conference on Osteoporosis, elaborado pela Sociedade } \\
\text { Canadense de Ginecologia e Obstetrícia , e o Scottish Intercollegiate } \\
\text { Guidelines Network (SIGN) são recuperados buscando-se "Osteoporosis" e } \\
\text { "Men" e nenhum com a adição do termo "Diagnosis". }\end{array}$ \\
\hline NICE (NHS) & $\begin{array}{l}\text { O National Institute of Clinical Evidence do Sistema Nacional de Saúde } \\
\text { britânico tem um guideline geral sobre osteoporose que se encontra em } \\
\text { formulação. }\end{array}$ \\
\hline Diretrizes AMB/CFM & $\begin{array}{l}\text { Guideline de tratamento da Osteoporose na pós menopausa (em atualização) } \\
\text { CMA Infobase }\end{array}$ \\
$\begin{array}{l}\text { "Osteoporosis" e "Men" não recupera guidelines. "Osteoporosis" recupera } \\
\text { nove, dos quais todos exceto dois abordam aspectos específicos às mulheres } \\
\text { ou tratamentos específicos (como o paratormônio). Novamente o Canadian } \\
\text { Consensus Conference on Osteoporosis, (2006) e o Clinical Practice } \\
\text { Guidelines for the diagnosis and management of osteoporosis in Canadá, } \\
\text { (2002, revisado em 04/07/2006), da Osteoporosis Society of Canadá. }\end{array}$
\end{tabular}

NGC

Recupera 7 guidelines que possam ter alguma informação relevante:

1. Diagnosis of osteoporosis in men, premenopausal woman, and children

(Sociedade Internacional de Desintometria Clínica). NGC:412

2. Diagnosis and treatment of osteoporosis (Institute for Clinical Systems

Improvement (ICSI). NGC:5146

3. Osteoporosis. NGC:2661

4. Management of osteoporosis. A national clinical guideline. NGC:3085

5. American Association of Clinical Endocrinologists medical guidelines for clinical practice for the prevention and treatment of postmenopausal osteoporosis: 2001 edition, with selected updates for 2003. NGC:3185 6. Prevention of hip fracture amongst people aged 65 years and over. NGC:3271

7. Summary of recommendations for clinical preventive services. NGC:5335

USPSTF e CTFPHC A análise de evidências se restringe a mulheres em Pós-menopausa.

Dentre as Sociedades de especialidade nacionais
SBMFC e SBCM não têm conteúdo específico sobre osteoporose. A SBGG em sua parte de Diretrizes tem um guideline sobre prevenção de quedas (Quedas em Idosos. Sociedade Brasileira de Geriatria e Gerontologia Elaboração Final: 16 de junho de 2001), e links para alguns artigos em seção específica dos quais um discute a mortalidade relacionada a Osteoporose em homens: Lois E. Wehren, M.D. Geriatric Times July/August 2002 Vol. III Issue 4 Osteoporosis Increases Mortality Risk in Men. A SOBRAO traz um informe sobre o tema, e link para um dos guindelines já recuperados previamente.

Dentre as Sociedades de especialidade internacionais
National Osteoporosis Foundation: páginas on line com alguns posicionamentos. Uma seção sobre homens voltada para Leigos, e um guideline $(19,20,23)$.

Osteoporosis Society of Canadá: recupera uma revisão da Radiologia, alguns guidelines inespecificos e um artigo: Management of osteoporosis in men: an update and case example. 
Fatores de risco:

O objetivo da triagem é a prevenção e avaliação do risco de fraturas ${ }^{16}$, comuns em osteoporose, com alta morbidade, que costumam ocorrer em média 10 anos mais tarde que em mulheres ${ }^{17}$. Homens que apresentam fraturas de quadril tendem a ser mais doentes, com maior risco de complicações pós-fratura e maior mortalidade que mulheres $^{17,18}$.

Dados na literatura sobre osteoporose e suas fraturas em homens são ainda limitados ${ }^{17}$. Devem-se discutir fatores de risco, especialmente exercício físico e cessação do tabagismo. Não há evidências claras de que exercício físico se relacione diretamente com a redução de danos na osteoporose, mas guidelines o recomendam fundamentados em benefícios globais ${ }^{17,18,19,20}$. A prevenção de quedas tem relação direta com a redução de fraturas ${ }^{18,20,21}$, sendo importante discutir riscos caseiros (como presença de tapetes) e evitar medicações que possam ocasionar sedação e tontei$\mathrm{ra}^{20}$.

A história familiar se refere a casos de osteoporose em pais e irmãos do paciente ${ }^{18}$. $\mathrm{Na}$ história pessoal pregressa, fratura vertebral ou de fragilidade aumentam marcadamente o risco de futuras fraturas ${ }^{16}$, devendo-se pesquisar ainda cifose e fraturas traumáticas antes dos $50 \operatorname{anos}^{18}$. Embora caucasianos sejam mais propensos ao quadro ${ }^{18}$, todos os grupos étnicos desenvolvem osteoporose ${ }^{20}$.

A história alimentar se confunde com as recomendações para pacientes com a doença estabelecida. Vários guidelines abordam ingestão de cálcio que varia de $1 \mathrm{~g} / \mathrm{dia}^{16,21}$ a $1200-1500 \mathrm{mg} / \mathrm{dia}^{17,21}$. Vitamina D deve ser reposta quando a exposição ao sol é inferior a 10 minutos por dia, com $400 \mathrm{UI} / \mathrm{dia}$, e não mais que $800 \mathrm{UI} / \mathrm{dia}^{20}$ sendo que os idosos necessitam de dosagens diárias de vitamina $\mathrm{D}$ de 700-800 17,20,21. Cafeína tem papel controverso: alguns guidelines a incluem como fator de risco ${ }^{16}$, outros advogam que não há evidências suficientes ${ }^{18}$. Não há evidências de que a fluoração da água altere o risco de osteoporose ${ }^{18}$.

Além de fatores similares àqueles das mulheres ${ }^{17}$, há outros preponderantes no gênero masculino ${ }^{20}$. Aproximadamente $50-60 \%$ de homens diagnosticados têm con- dições clínicas que podem levar à perda óssea ${ }^{19}$ tais como exposição a esteroides, anticonvulsivantes, alguns quimioterápicos e antiácidos que contenham alumínio; doenças crônicas que afetem rins, pulmões, estômago e intestinos. Doenças que alterem níveis hormonais como hiperparatireoidismo, hipogonadismo, que pode apresentar-se assintomático, e baixos níveis de testosterona, enfim, aqueles relacionados a hábitos de vida, tais como tagabismo, etilismo, baixa ingestão de cálcio, exercício físico inadequado e baixo peso (especialmente perda de peso recente) ${ }^{19,20}$. Até um terço de idosos com osteoporose têm baixos níveis de testosterona ${ }^{17}$, mas grande número de homens com fraturas ou baixa densidade mineral não têm causa específica isolada, definindo o quadro de osteoporose idiopática ${ }^{19}$.

São classificados de baixo risco para baixa DMO, e inicialmente não candidatos a densitometria ${ }^{21}$ homens eugonadais sem história de fratura de baixo impacto que não estejam em corticoterapia e sem história de doenças crônicas relacionadas com osteopenia ${ }^{1}$. São explicitados os fatores de risco maiores e menores para a doença (Tabela 1).

\section{Diagnóstico:}

A avaliação é similar à realizada nas mulheres. Fraturas que ocorrem na ausência de trauma são suspeitas de osteoporose, especialmente em homens sem fatores de ris$\mathrm{co}^{19}$. A medida da DMO pela densitometria é o melhor preditor isolado do risco de fraturas ${ }^{21}$. Radiografias simples não excluem ou diagnosticam osteoporose, e se há osteopenia grave, deve-se realizar densitometria ${ }^{18}$.

Definir osteoporose pela presença de fraturas de baixo impacto é controverso. Alguns guidelines definem fraturas de fragilidade como diagnósticas ${ }^{21,22}$ e advogam que mulheres acima de 80 anos com múltiplas fraturas vertebrais deveriam ser submetidas diretamente a terapia medicamentosa $^{18}$, e que se deveria iniciar terapia em mulheres com uma fratura prévia vertebral ou do quadril ${ }^{23}$. Fraturas, especialmente em membros, causam acelerada perda óssea, não completamente reversível, e podem causar fraturas subsequentes, além de possíveis "influências mecânicas causadas por haver uma fratura que aumente os riscos subsequentes de alteração do equilíbrio e risco aumentado de queda"21. 
Tabela 1. Fatores de risco para baixa densidade óssea e futuras fraturas que identificam quem deveria ser triado para osteoporose.

\begin{tabular}{|c|c|}
\hline Fatores de Risco Maiores $^{a}$ & Fatores de Risco Menores \\
\hline $\begin{array}{l}\text { Fratura de fragilidade com trauma mínimo } \\
\text { após os } 40 \text { anos (queda da altura ou menos) } \\
\text { ou de compressão vertebral. } \\
\text { Corticoterapia por } 3 \text { meses em dos } \geq 5 \mathrm{mg} \\
\text { prednisoma / dia b } \\
\text { Osteopenia radiográfica ou deformidade } \\
\text { vertebral, consistentes com fratura b,c } \\
\text { História familiar de fratura osteoporótica } \\
\text { Síndrome de má-absorção } \\
\text { Hiperparatireoidismo primário } \\
\text { Propensão para quedas } \\
\text { Hipogonadismo } \geq 5 \text { anos } \\
\text { Perda de mobilidade prolongada ( }>\text { um ano) } \\
\text { Transplantado de órgão sólido ou medula óssea } \\
\text { Medicamentos para tumores malígnos e Doenças } \\
\text { crônicas sabidamente associadas a perda óssea. }\end{array}$ & $\begin{array}{l}\text { Artrite Reumatóide } \\
\text { História pregressa de hipertireoidismo clínico } \\
\text { Terapia anticonvulsivante crônica } \\
\text { Baixa ingestão de Cálcio } \\
\text { Fumante } \\
\text { Uso excessivo de álcool } \\
\text { Uso excessivo de cafeína (controverso) } \\
\text { Peso abaixo de } 57 \mathrm{~kg} \text {, ou abaixo IMC } \\
\text { Perda de } 10 \% \text { do peso em relação aos valores } \\
\text { aos } 25 \text { anos } \\
\text { Terapia crônica com heparina }\end{array}$ \\
\hline
\end{tabular}

Fontes: Brown e Fortier ${ }^{16}, \mathrm{SIGN}^{18}$, ICSI ${ }^{21}$.

Em última análise, deve-se ao menos considerar homens com fraturas de baixo impacto como candidatos para trata- mento farmacológico e fisioterapia ${ }^{21}$ e adultos com história de fratura vertebral, do quadril ou do antebraço em risco aumentado para fraturas ${ }^{21}$. Fraturas de fragilidade, ou de baixo impacto, são aquelas resultantes de tosse, espirros ou movimentos abruptos sem trauma, fraturas por "quedas da própria altura" e fraturas de compressão vertebral documentada radiologicamente a despeito de sintomas ${ }^{21}$.

\section{"Low-impact fracture defines osteoporosis and requires therapy" 21}

Sociedades de Densitometria apontam uma suposta necessidade do exame como único método diagnóstico

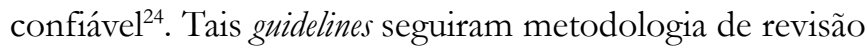
por pares, com base em consensos de especialistas, em que não se especifica qual a fonte de evidências nem os artigos consultados e, portanto, deve ser vista com cautela.

A tecnologia de escolha para densitometria apontada por todos os guidelines é a DEXA (dual-energy X-ray absorptiometry) da coluna lombar ou quadril ${ }^{18,22,24}$. Porém, somente deve ser realizada em pacientes que estejam dispostos a realizar o tratamento ${ }^{21}$. A classificação apresentada pela OMS não se aplica a homens de modo similar às mulheres $^{18}$. Há necessidade de se criar um banco de dados masculino para o escore $\mathrm{T}$ de referência. Sendo assim, recomenda-se ${ }^{24}$ :

- Homens com 65 anos ou mais têm osteoporose se Tscore $<-2.5$ ou menor em um banco de dados masculino. - Entre 50 e 65 anos, a osteoporose é diagnosticada se Tscore $<-2.5$ em um banco de dados masculino se há fatores de risco para fraturas

- Homens com causas secundárias (corticoterapia, hipogonadismo, hiperparatireoidismo) são diagnosticados se há baixa DMO. Estes pacientes podem ter um risco para fraturas diferente com valores dados de DMO.

- O diagnóstico de osteoporose em homens abaixo de 50 anos não é fundamentado exclusivamente em critérios da densitometria.

Um guideline de Singapura sugere uma avaliação de risco usando o OSTA (Osteoporosis Self-assessment Tool for Asians) como filtro inicial. Questionários como este são citados em alguns guidelines, porém serão recuperados em 
maior magnitude no passo 4 desta revisão (vide a se- guir) ${ }^{22}$.

Tratamento:

Ao diagnóstico de Osteoporose, a avaliação inclui pesquisa de fatores de base responsáveis pela perda óssea, inclusive exames bioquímicos e ocasionalmente biópsia óssea, e avaliação clínica adequada para identificar a existência de condições médicas ou medicamentos que contribuam para a perda de equilíbrio e risco de quedas ${ }^{19}$.

A indicação de drogas é individualizada pela idade, $\mathrm{DMO}$, risco de fratura e queda ou perda óssea. A escolha depende de quais estão disponíveis e seus efeitos colaterais. Sugere-se que homens e outros grupos que não mulheres na pós-menopausa sejam avaliados em clínicas de osteopo$\operatorname{rose}^{22}$.

A maioria das revisões foca em mulheres, e neste há boas evidências de que o uso de bifosfonados, como o Alendronato, e Cálcio reduzem o risco de fraturas ${ }^{23,25}$. Em homens e mulheres em corticoterapia, Alendronato e Risendronato melhoram massa óssea em quadril e coluna, independentemente de dose ou duração, bem como em doença de base ou massa óssea lombar basal. Estudos com Risendronato e Alendronato demonstraram ainda redução na incidência de fraturas vertebrais ${ }^{17}$.

Para saber mais sobre efeitos colaterais das medicações sugere-se ler: Diagnosis and Treatment of Osteoporosis - Institute for Clinical Systems Improvement ${ }^{21}$ que conta com uma sessão específica de medicação e efeitos colaterais.

Em homens osteoporóticos, o Alendronato aumenta a DMO em coluna, quadril e total, prevenindo fraturas vertebrais e redução de peso, com estudos de nível I suportando seu uso para prevenir a perda óssea masculina ${ }^{17,21}$. Não foram demonstradas reduções no risco de fraturas não-vertebrais, devido ao tamanho das amostras estudadas ${ }^{17}$.

O tratamento consiste inicialmente em identificar e tratar causas específicas de perda óssea e manutenção de dieta balanceada com consumo adequado de cálcio (1.000 $\mathrm{mg} / \mathrm{dia}$ em homens jovens e $1.200-1.500 \mathrm{mg} / \mathrm{dia}$ naqueles acima de 65 anos) e vitamina D (400-800 IU/dia), evitando inatividade, com exercícios (especialmente com pesos e treinamento de resistência) ${ }^{18,19,20}$. Outros tratamentos incluem calcitonina, hormônio da tireoide, dentre outros, sem informações suficientes sobre eficácia ${ }^{19}$.

A reabilitação do paciente com osteoporose é tratada em um artigo específico da National Osteoporosis Foundation ${ }^{26}$.

Acompanhamento do tratamento:

A DMO não é indicador de sucesso do tratamento, pois muitas vezes o tratamento não leva ao aumento dos índices de massa óssea. Embora represente a medida mais acurada e precisa da densidade minera ${ }^{16}$, não é uma indicação consensual, com referência de que somente uma avaliação da DMO seria necessária ${ }^{18}$, havendo necessidade de uma segunda densitometria se ela realmente puder modificar o tratamento (suspeita de não adesão ou de falha terapêutica). Apesar disso, outros consensos definem a medida da DMO por DEXA como o melhor parâmetro de monitorização da resposta ao tratamento ${ }^{21}$.

Os marcadores de renovação óssea podem ser utilizados para medir aderência ao tratamento ou efetividade dos fármacos ${ }^{16}$.

Pacientes com uma ou mais fraturas de fragilidade são alvos prioritários para esta propedêutica ${ }^{18}$. Outros exames podem fazer parte de uma propedêutica complementar, de acordo com a suspeita clínica de doença subjacente ${ }^{21}$.

Ainda dentro do passo 3, foram realizadas buscas nos sítios das principais sociedades de especialidades, que não encontraram artigos sobre o tema na Sociedade Brasileira de Medicina de Família e Comunidade e na de Clínica Médica. Três artigos foram recuperados no sítio da Sociedade Brasileira de Geriatria e Gerontologia, e um guideline na NOF.

Passo 4. Localização de revisões sistemáticas pela opção Clinical Queries e pela Colaboração Cochrane.

- PubMed Clinical Queries - http://www.ncbi.nlm.nih.gov/ entrez/query/static/clinical.html.

- Cochrane/Bireme-http://cochrane.bireme.br/cochrane/ main.

A maior dificuldade do Pubmed Clinical Queryes (e da base de dados do Medline como um todo) é que as principais revistas que abordam Osteoporose - J. Clin. Densitometry, Osteoporos Intl, Bone - estão indisponíveis em livre 
acesso. Foram recuperados 91 artigos, porém a maioria é descartada já no título.

Uma meta-análise ${ }^{27}$ determinou sensibilidade e especificidade do Ultrassom de Calcâneo Quantitativo (USC) frente a densitometria em 2006. Para o escore -1 ao USC, a sensibilidade atingiu 79\% (95\% CI, 69\% to 86\%) e a especificidade foi 58\% (CI, 44\% to 70\%) em relação a T-scores $<-2.5$ à DEXA em quadril e coluna. Para um escore de 0 no USC, a sensibilidade aumentou para 93\% (IC $=87 \%$ to $97 \%$ ), mas com especificidade de $24 \%$ (IC $=10 \%$ to $47 \%$ ). $\mathrm{O}$ pequeno número de estudos prejudicou a avaliação da acurácia diagnóstica do USC, e os resultados do USC nos pontos de corte avaliados não excluem ou confirmam a osteoporose diagnosticada pela DEXA. Seriam necessários mais estudos antes de o USC ser recomendado mesmo como screening para Osteoporose.

Gemalmaz \& $\operatorname{cols}^{28}$ estudaram o USC no âmbito da APS, comparando-o com dados antropométricos e de estilo de vida, com sensibilidade de 73,7\% (especificidade $57,4 \%$ ) para identificar pacientes osteoporóticos. Houve correlação fraca entre o USC e os T-scores da densitometria na coluna lombar $(r=0.310, \mathrm{P}<0.001)$ e no colo do fêmur $(\mathrm{r}=0.288, \mathrm{P}<0.001)$. A faixa de idade de melhor correlação entre USC e DEXA foi 50-59 anos. Mesmo com acurácia baixa, o USC teria potencial para definir pacientes com necessidades de prevenção de fraturas em países em desenvolvimento e áreas rurais.

Sinnott \& $\operatorname{cols}^{29}$ estudaram ferramentas de triagem (USC, a fórmula Osteoporosis Self-assessment Tool - OST, "weight-based criterion" - WBC e Índice de Massa Corpórea - IMC) para homens afro-americanos, demonstrando sensibilidade de $83 \%$ e especificidade de $71 \%$ para o USC, sensibilidade de $83 \%$ (especificidade $57 \%$ ) para OST no ponto de corte 4,0, e baixa sensibilidade para WBC $(74 \%$, especificidade $50 \%)$ e IMC (83\%, especificidade $43 \%)$. A OST baseia-se em cálculo de idade e peso, sendo um indicador com maior facilidade de uso na prática clínica.

Castrillón \& $\operatorname{cols}^{30}$ desenvolveram em Hospital Universitário de Valladolid (Espanha), publicado no J Clin Densitometry, avaliação de OST + USC, usando pontos de corte de 3,0 para homens, com sensibilidade da OST isoladamente de apenas 39\% (especificidade de $86 \%$ ), enquanto em mulheres encontrou-se sensibilidade de $94 \%$ e especificidade de $59 \%$ para o ponto de corte 2,0 . O uso do USC não alterou significativamente estes valores em homens.

Gudmundsdottir \& $\mathrm{Cols}^{31}$, em estudo populacional islandês, sugerem diferentes pontos de corte para o USC: usando o ponto de corte $-0,5$ em homens, e concomitantemente os valores de ponto de corte inferiores a -1.0 para mulheres entre 50 e 65 anos e $-2,5$ para mulheres mais velhas, exclui-se confiavelmente de $30-40 \%$ da população "saudável" de um screening pela DEXA. Para pacientes com fraturas osteoporóticas haveria a necessidade de estudos específicos para determinar o valor do USC.

Shepherd \& cols desenvolveram um questionário de avaliação de risco de osteoporose em homens (MORES) para APS considerando idade, peso e históricode doença pulmonar obstrutiva crônica (DPOC), com sensibilidade de 0,93 (IC 95\%: 0,85-0,97) e especificidade de 0,59 (IC 95\%: 0,56-0,62) para um escore final de 6,0 em população americana, especialmente em homens acima de 60 anos, a partir de base populacional de 2.995 homens acima de 50 anos no National Health and Nutrition Examination Survey (NHANES) III submetidos a DEXA. Seriam necessárias 279 triagens em homens para evitar uma fratura de quadril em 10 anos (NNS) ${ }^{32}$.

Estudo de validação da escala OST na Bélgica comparada a outros escores (SCORE, ORAI, OSIRIS) aponta a OST como de melhor sensibilidade e menor especificidade em relação aos demais. $\mathrm{O}$ ponto de corte OST $<2$ como método pré-triagem à avaliação da DMO traz sensibilidade de $85 \%$ na coluna lombar e $97 \%$ no quadril para um T-score $<2.5$ na densitometria, e especificidade de 34\% no quadril a $37 \%$ no colo do fêmur e coluna lombar. $\mathrm{O}$ valor preditivo negativo (VPN) foi alto em todos os sítios tios (89-99\%), demonstrando a utilidade do OST para identificar pacientes que tenham densidade óssea normal e não deveriam se submeter a densitometria ${ }^{33}$.

Ghazi e colaboradores ${ }^{34}$ estudaram OST em homens brancos de Marrocos em ambulatório de especiali- 
dade e encontraram VPN $=87-98 \%$, demonstrando a utilidade de OST (ponto de corte $<2,0$ ) para pacientes com DMO normal, que não deveriam realizar densitometria. Sua sensibilidade variou de 63\% (especificidade 58\%) em relação à densitometria da coluna lombar a 87\% (especificidade 59\%) em relação à densitometria do quadril.

Skedros, Sybrowsky e Stoddart estudaram OST ${ }^{35}$ para identificar homens sob risco de alteração da DMO em comparação a um questionário de fatores de risco de um serviço de ortopedia. Encontraram sensibilidade de $85 \%$, especificidade de $64 \%$, VPP de $31 \%$, e VPN de $96 \%$ para um ponto de corte $<2,0$.

$\mathrm{Na}$ biblioteca Cochrane, utilizando-se os termos senile osteoporosis and diagnosis foram recuperadas 10 revisões, entretanto nenhuma delas se referia diretamente ao diagnóstico de osteoporose. Quando realizada revisão com o unitermo age-related osteporosis, não foram recuperados artigos.

Passo 5. Para assegurarmos que a revisão realizada encontra-se atualizada, deve ser completada a busca, utilizando artigos originais recentes:

- PubMed http://www.ncbi.nlm.nih.gov/sites/entrez (Foram selecionados os "Limits": "published in the last: 2 years" e gender="male", conforme na figura \#\#): traz 259 artigos, porém após estabelecer os critérios de leitura, somente dois artigos de interesse são selecionados.

- Bireme/BVS - http:/ /www.bireme.br, usando os termos "osteoporose", "diagnóstico" e "homem" recupera seis artigos no LILACS, sendo dois artigos de opinião sobre o tema publicados na Revista Brasileira de Endocrinologia e Metabologia, e quatro artigos no SciELO.

- DARE - http://www.york.ac.uk/inst/crd/.

- American College of Physicians (ACP) Journal Club http://www.acponline.org/journals/acpjc/jcmenu.htm.

Nos passos 4 e 5, é importante estabelecer uma leitura sistemática: iniciar sempre pelo título do artigo, que, se não contiver a questão levantada, por mais interessante que possa parecer, deve ser descartado. Estudos em animais podem ser promissores, mas também não respondem a uma dúvida da prática clínica.

O título do artigo servirá para descartar a maior parte dos artigos em questão. Caso o tema seja compatível com a questão, ou caso ainda existam dúvidas, o resumo será esclarecedor para avaliar se tal artigo responde à pergunta clínica. Neste ponto, já há um número de artigos restrito o suficiente para se dedicar a uma leitura mais detalhada.

O próximo ponto são as referências bibliográficas do artigo, para avaliar se o trabalho se baseia em parâmetros confiáveis, e logo a seguir, nesta ordem, introdução e conclusão. Até este ponto já é possível definir a utilidade do artigo para a prática clínica.

Dentre os poucos artigos recuperados no passo 5, excetuando-se revisões e ensaios clínicos já recuperados pelo passo 4, destaca-se estudo da relação custo-efetividade da abordagem da osteoporose em idosos pela densitometria e tratamento ${ }^{36}$. Em homens acima de 65 anos com relato de fratura, e homens acima de 80 anos sem fratura, "densitometria seguida de tratamento" pode ser custo-efetiva. $\mathrm{Na}$ faixa de 70 anos sem relato de fraturas, o custo-efetividade dependerá do custo do tratamento (se abaixo de 500 dólares) e do custo dos anos de vida perdidos ajustados à idade (se acima de 100.000 dólares).

\section{Discussão}

Cumpridas as etapas I e II, representadas pelos passos 1 a 5 , procede-se às etapas relacionadas à avaliação da informação recuperada?

III. A análise qualitativa da evidência obtida aponta guidelines fundamentados em evidências, e artigos com Nível 1 e 2 de evidência que, embora com alguns viéses, trouxeram a melhor informação disponível para a abordagem do paciente em questão.

IV. Análise quantitativa da evidência aponta número de artigos sobre o diagnóstico da osteoporose menor que em mulheres, mas foram recuperados estudos - especialmente meta-análises - que abordaram número suficiente de pacientes para obter conclusões relevantes quanto ao diagnóstico e à triagem da doença em homens.

V. A análise de custo-efetividade aponta a responsabilização do paciente na abordagem direta de seu quadro clínico.

Na prática, a densitometria não está disponível na 


\section{MORES}

\begin{tabular}{lc}
\multicolumn{1}{c}{ Fator } & Score \\
\hline $\begin{array}{l}\text { Idade } \\
\leq 55 \text { anos }\end{array}$ & 0 \\
$56-74$ anos & 3 \\
$\geq 75$ anos & 4 \\
Peso & \\
$\leq 70 \mathrm{~kg}(\leq 154 \mathrm{lb})$ & 6 \\
$>70-80 \mathrm{~kg}(>154-176 \mathrm{lb})$ & 4 \\
$>80 \mathrm{~kg}(>176 \mathrm{lb})$ & 0 \\
DPOC & 3 \\
\hline
\end{tabular}

Ponto de corte: $>6$ para MORES

\section{SCORE}

\begin{tabular}{ll}
\hline \multicolumn{1}{c}{ Fator } & \multicolumn{1}{c}{ Score } \\
\hline Raça não-negra & +5 \\
Artrite Reumatóide & +4 \\
Fratura não-traumatica após 45 anos & +4 por fratura, máximo de 12 pontos \\
Idade & +3 para cada década \\
Terapia hormonal & +1 se nunca \\
Peso & -1 para cada $10 \mathrm{lb}(4.5 \mathrm{~kg})$ \\
\hline
\end{tabular}

Ponto de corte: $>7$ para SCORE

ORAI

\begin{tabular}{lll}
\hline \multicolumn{1}{c}{ Fator } & \multicolumn{1}{c}{ Score } \\
\hline Idade $>75$ anos & +15 \\
Idade 65 - 74 anos & +9 & +5 \\
Idade 55 - 64 anos & +9 & +3 \\
Peso $<60 \mathrm{~kg}$ & +2 se não estiver em uso de estrógeno \\
Peso 60 - 70 kg &
\end{tabular}

Ponto de corte: $>8$ para ORAI 


\section{OSIRIS}

\begin{tabular}{ll}
\hline \multicolumn{1}{c}{ Fator } & \multicolumn{1}{c}{ Score } \\
\hline Peso (kg) & $+0.2 \mathrm{X}$ peso \\
Idade (anos) & $-0.2 \mathrm{X}$ idade \\
História de fratura(s) de baixo impacto & -2 \\
Terapia hormonal & +2 \\
\hline Ponto de corte: $<1$ para OSIRIS &
\end{tabular}

\section{OST}

\begin{tabular}{cc}
\hline Fator & Score \\
\hline Peso (kg) & $0.2 \mathrm{X}$ (peso - idade) \\
Idade (anos) & \\
\hline Ponto de corte: $<2$ para OST
\end{tabular}

Ponto de corte: $<2$ para OST

rede pública, cabendo ao paciente arcar com o exame. Já quanto ao tratamento, a rede pública estadual de Minas Gerais tem como fornecer a medicação necessária para seu tratamento mediante densitometria.

VI. Avaliação da aplicabilidade dos dados para a prática clínica: a osteoporose é um problema de saúde mundial, e as evidências apontam uma situação que dependerá diretamente da vulnerabilidade econômica e social do paciente.

A osteoporose é um problema de saúde em homens, principalmente caucasianos, acima de 60 anos, com risco de fraturas de $29 \%$ ao longo da vida. A decisão clínica vai ser amparada no custo-efetividade do diagnóstico e da intervenção terapêutica, com base na melhor evidência disponível. A falta de consensos em homens com osteoporose reflete em baixas taxas de intervenção clínica. As evidências apontam para a necessidade da densitometria óssea, padrão-ouro para o diagnóstico e considerada por alguns indispensável para o diagnóstico clínico. As evidências ainda não apontam outros métodos, embora alguns guidelines definam fraturas de fragilidade como diagnósticas de osteoporose.

No setor público municipal de saúde, o exame tem difícil acesso. Em pesquisa telefônica informal na rede pública de três centros urbanos da região metropolitana, somente o município de Belo Horizonte disponibiliza densitometria, desde setembro de 2007, mediante consulta com subespecialistas. A rede hospitalar do estado de Minas Gerais também não disponibiliza o exame, segundo informações coletadas na rede FHEMIG, e a Secretaria Estadual de Saúde de Minas Gerais fornece medicamentos para o tratamento da osteoporose mediante o resultado da densitometria.

As evidências não suportam o uso do USC como exame diagnóstico de osteoporose, embora com potencial de uso como triagem para a densitometria (aumentando a probabilidade pré-teste). Seu uso como possível exame diagnóstico demanda parâmetros quantitativos confiáveis.

Questionários de avaliação de fatores de risco para osteoporose não têm valor diagnostico, somente como triagem para a realização da densitometria. A ferramenta OST, também utilizada como método de triagem, tem boa confiabilidade, embora alguns estudos apresentem resultados controversos de sensibilidade e especificidade em relação a DEXA. Questionários de pré-triagem para osteoporose, embora sensíveis, são pouco específicos e servem mais para estratificar o risco entre os pacientes que realmente afastar a necessidade da densitometria. Em sua maioria não alteram a recomendação de realizar densitometria em mulheres acima de 65 anos.

Outro ponto a se considerar é o custo do diagnóstico e tratamento. Estudo de custo-efetividade aponta que o diagnóstico e tratamento em homens somente seria custoefetivo com um gasto de seguridade social calculado por Anos de Vida Ajustados à Qualidade (QALY) de 100.000 dólares, e o custo do tratamento com bifosfonados orais abaixo de 500dólares/ano para homens acima de 70 anos sem relato de fraturas. Quando há história de fratura prévia associada, a relação custo-efetividade é positiva para homens acima de 65 anos $^{23}$. O custo de fraturas osteoporóticas anuais para o sistema de saúde estadunidense foi estimado em 17 bilhões de dólares, e o custo de uma simples fratura de quadril, estimada em 40.000 dólares em 2001. Estima- tivas apontam que o número de fraturas de quadril podem mais 
que triplicar até o ano $2040^{36}$.

Estudo brasileiro sugere a suplementação universal de cálcio e vitamina $\mathrm{D}$ para idosos, com menor custo e efetividade semelhante à triagem para osteoporose e tratamento. Em nosso país este custo seria superior a 10.000 reais, contra um custo de tratamento da fratura estabelecido em 2.000 reais $^{37}$. Uma meta-análise aponta o uso de cálcio com ou sem vitamina D para o prevenir da osteoporose em pessoas acima de 50 anos, com doses de $1.200 \mathrm{mg}$ de cálcio e 800 IU de vitamina D. Os dados foram coletados a partir de 29 estudos randomizados ( $n=63$ 897) com pessoas acima de 50 anos, e como desfechos foram eleitos "fraturas" e "alterações percentuais de densidade mineral". Em estudos que reportaram "fraturas" como desfecho (17 trials, $n=52$ 625), a suplementação obteve redução de $12 \%$ no risco de fraturas de qualquer tipo. (RR 0,88; IC 95\%: 0,83-0,95; $\mathrm{p}=0,0004)$. A redução do risco de fraturas foi maior $(24 \%)$ em estudos nos quais a taxa de adesão foi alta $(\mathrm{p}<0,0001)$. Em estudos que reportaram densidade mineral como desfecho (23 trials, $n=41.419)$, houve redução de perda óssea de 0,54\% (IC 0,35-0,73; p<0,0001) no quadril e 1,19\% (IC 0,76-1,61\%; $\mathrm{p}<0,0001$ ) na coluna. O efeito do tratamento foi melhor com as doses de cálcio de 1.200 mg ou mais que em taxas menores $(0,80$ vs 0,$94 ; \mathrm{p}=0,006)$, e com doses de vitamina D de $800 \mathrm{IU}$ ou mais que com doses menores $(0,84 \text { vs } 0,87 ; \mathrm{p}=0,03)^{38}$.

A introdução de tratamento e métodos diagnósticos para osteoporose tem outro dificultador: a saúde pública brasileira lida com prioridades de métodos diagnósticos e de tratamento de doenças que talvez tenham custo-efetividade e custo-eficácia ainda melhores que a abordagem da osteoporose, que podem melhorar a sobrevida e a qualidade de vida de um número maior de pessoas, ou ter maior impacto na redução da mortalidade ou morbidade de populações.

Alguns consensos apontam a possibilidade de tratamento sem densitometria em mulheres com fraturas patológicas. Considerando-se fraturas de fragilidade diagnósticas de osteoporose e a dificuldade de acesso à densitometria, pode-se aventar a possibilidade de se realizar tratamento para osteoporose a partir de uma fratura de fragilidade, pesando sempre os riscos e benefícios do tratamento medicamentoso. $\mathrm{O}$ custo do tratamento anual no varejo oscila entre 693,57 reais e 1.588,55 reais (USD 346,78 a USD 794,27), segundo pesquisa informal de preços de medicamentos tomada em quatro grandes redes farmacêuticas. $\mathrm{O}$ custo da densitometria é de 160 a 170 reais, de acordo com dois centros de diagnóstico. Obviamente os valores para o tratamento e diagnóstico realizados a partir da rede pública de saúde teriam gastos diferenciados. Ou seja, mesmo optando pelo tratamento a partir de uma fratura de fragilidade, é necessário avaliar se o paciente estará disposto a arcar com o tratamento, e tal disposição é condição para realizar densitometria.

A metodologia de pesquisa proposta tem valor para dúvidas surgidas da prática clínica do Médico de Família e Comunidade. Tal metodologia é diferente, por exemplo, de uma revisão bibliográfica com fins científicos ou de pesquisa, que consideram as fontes primárias e a priori ignoram consensos e/ou guidelines.

As informações são classificadas em primárias pesquisas originais - e secundárias - avaliações críticas de um conjunto de informações primárias. São consideradas fontes confiáveis as fontes primárias - desde que avaliadas de maneira integrada - e as secundárias - desde que explicitado claramente o mecanismo de busca da informação. Uma meta-análise, por exemplo, é considerada uma fonte primária, pois há metodologia científica específica de busca, decomposição e reanálise estatística de um conjunto de dados.

Outras boas fontes de artigos científicos são alertas recebidos por e-mail. No caso da osteoporose em homens, alguns artigos relevantes foram inicialmente recuperados assim. São interessantes o Physician First Watch e o Alerta de pediódicos da WONCA, para a APS. Outra opção são alertas específicos que podem ser configurados no Medline. A partir de parâmetros de revisão, pode-se solicitar que todos os novos artigos que se encaixem em seus limites de pesquisa sejam enviados por e-mail semanalmente. 


\section{Conclusão}

Grandes estudos sistematizados e randomizados, bem como meta-análises, trabalham com pacientes livres de comorbidades, o que não representa a prática clínica cotidiana e, portanto, sua aplicabilidade demanda individualização e não exclui a experiência clínica acumulada. Em vista das recentes críticas à padronização de "pacientes" em estudos clínicos, e à pouca adequação da Medicina Baseada em Evidências à prática real, é uma boa conduta individualizar a evidência clínica para o paciente - Pacient Oriented Evidence that Matters (POEMS). Consiste em submeter a informação encontrada a três critérios:

- Responde uma questão que o médico encontra na prática clínica?

- Mede resultados que geram preocupação ao paciente: sintomas, morbidade, qualidade de vida e mortalidade?

- Tem o potencial de mudar o modo de prática do médico?

Nesta revisão, os dados recuperados são relevantes para o paciente, pois afetam sua qualidade de vida, morbidade, mortalidade, respondendo a uma dúvida da prática clínica, porém com dificuldades para sua aplicação no sistema público de saúde brasileiro, a chamada validade externa, tendo em vista as dificuldades de acesso à tecnologia proposta para o diagnóstico, e mesmo as opções de tratamento.

Evita-se indicar métodos diagnósticos rígidos ou abordagens padronizadas a todos os pacientes da rede pública, já que há necessidade de individualização do tratamento por outras comorbidades, polifarmácia, risco de quedas, poder aquisitivo, pretensão de se submeter ao tratamento, e especialmente com o quadro clínico estabelecido.

A osteoporose demanda exames e tratamentos não plenamente disponíveis na rede pública de saúde, e há a possibilidade de que a suplementação universal preventiva de cálcio de vitamina $\mathrm{D}$ sejam opções à realização de densitometria para todos os pacientes. Para o diagnóstico de osteoporose, e indicação de tratamento com bifosfonados (como alendronato) e cálcio + vitamina $\mathrm{D}$, a densitometria por DEXA continua sendo o exame de escolha, e necessário para indicação deste tratamento.
Em alguns casos, tais como a presença de fraturas de fragilidade, pode-se aventar a hipótese de tratamento a despeito da densitometria, porém o exame é a parte mais barata da abordagem. Mesmo porque, protelar o tratamento em pacientes com fraturas osteoporóticas torna-se uma opção não-isenta de riscos de graves complicações.

\section{Referências}

1. Savassi LCM. Bancos de dados em Atenção Primária a Saúde. AMMFC: Jornal Interativo. 2006; 3(4):3.

2. De-Sousa JC. Quantum Sufficit. Rev Port Clin Geral 2007;23:253-7

3. Bernardo WM, Cuce-Nobre MR, Jatene FB. A prática clínica baseada em evidências. Parte II - buscando as evidências em fontes de informação. Rev Assoc Med Bras. 2004; 50(1): 104-8.

4. Keren R, Chan E. A Meta-analysis of Randomized, Controlled Trials Comparing Short- and Long-Course Antibiotic Therapy for Urinary Tract Infections in Children. Pediatrics 2002;109(5). Disponível em: http:// www.pediatrics.org/cgi/content/ full/109/5/e70.

5. Oliveira ACD, Dias RB, Souza LC. Buscando Evidências em Fontes de Informação. AMMFC: MFC de A a Z. Disponível em: http://www.smmfc.org.br/busca_evid. htm Acesso em: set. 2007.

6. Velázquez Alberto. Introdução à epidemiologia clínica.In: PROFAM (programa de educação à distância de Medicina familiar e ambulatorial). [s.l.s.n.n.]; 2002. p. 87.

7. Duncan BB, Schmidt MI, Giugliani ERJ. Medicina Ambulatorial: condutas de atenção primária baseadas em evidências. 3. ed. Porto Alegre (RS): ARTMED; 2004.

8. Canela Cáceres JR, Louro González A. Dónde y cómo buscar la información necesaria. Disponível em: http:// www.fisterra.com/mbe/mbe_temas/13/buscar _informacion.htm Acesso em: 23/11/2004.

9. Chaves SR. Medicina baseada em evidências e decisão terapêutica. In: De-Pinho H. Medicina e Saúde da Família - Diagnóstico, Tratamento e Prática Ambulatorial. Rio de Janeiro: Medbook; 2007.

10. Cuce Nobre MR, Bernardo WM, Jatene FB. A prática 
clínica baseada em evidências. Parte I - questões clínicas bem construídas. Rev Assoc Med Bras. 2003; 49(4): 445-49.

11. Lopes AA. Medicina Baseada em Evidências: a arte de aplicar o conhecimento científico na prática clínica. Rev Ass Med Brasil. 2000; 46(3): 285-8.

12. Finkelstein Joel S. Osteoporose. In: Goldman L, Ausiello D. Cecil Tratado de Medicina Interna. Rio de Janeiro: Elsevier; 2005. p. 1804-1812.

13. Pereira SRM, Mendonça LMC. Osteoporose e osteomalácia. In: Freitas EV, Py L, Cançado F, Doll J, Gorzoni ML. Tratado de Geriatria e Gerontologia. Rio de Janeiro: Guanabara Koogan; 2006. p. 798-813.

14. Descritores em Ciências da Saúde (DeCS). Osteoporose. Disponível em: http://decs.bvs.br/ Acesso em: set. 2007. 15. Medical Subject Heading (MeSH). Osteoporosis. Disponível em: http://www.ncbi.nlm.nih.gov/mesh/ Acesso em: set. 2007.

16. Brown JP, Fortier M, Society of Obstetricians and Gynaecologists of Canada (SOGC). Canadian Consensus Conference on Osteoporosis, JOGC. feb. 2006; S95-S112. 17. University of Michigan Health System. Osteoporosis: prevention and treatment. Ann Arbor (MI): University of Michigan Health System. Jul; 2005. 13 p.

18. Scottish Intercollegiate Guidelines Network (SIGN). Management of osteoporosis. A national clinical guideline. Edinburgh: Royal College of Physicians; 2003. 45 p. Disponível em: http://www.sign.ac.uk

19. National Osteoporosis Foundation (NOF). Strategies for Osteoporosis. Disponível em http://www.nof.org/ men/strategies_men.htm Acesso em: ago. 2007.

20. National Osteoporosis Foundation (NOF). Osteoporosis and Men. Disponível em http://www.nof .org/men/index.htm Acesso em: ago. 2007.

21. Institute for Clinical Systems Improvement (ICSI). Diagnosis and treatment of osteoporosis. Bloomington (MN): Institute for Clinical Systems Improvement (ICSI). Jul; 2006. 64 p.

22. Singapore Ministry of Health. Osteoporosis. Singapore: Singapore Ministry of Health. Feb; 2002. 63 p.

23. National Osteoporosis Foundation (NOF). Physician's guide to prevention and treatment of osteoporosis. Washington (DC): National Osteoporosis Foundation. Apr.; 2003. 37p.

24. Institute for Clinical Systems Improvement (ICSI). Diagnosis and treatment of osteoporosis. Bloomington (MN): Institute for Clinical Systems Improvement (ICSI). Jul; 2006. $64 \mathrm{p}$.

25. New Zealand Guidelines Group (NZGG). Prevention of hip fracture amongst people aged 65 years and over. Wellington (NZ): New Zealand Guidelines Group (NZGG). Jun; 2003. 31p.

26. National Osteoporosis Foundation (NOF). Health professional's guide to rehabilitation of the patient with osteoporosis. Washington (DC): National Osteoporosis Foundation; 2003. 31p.27. Nayak S, Olkin I, Liu Hau, Grabe M, Gould MK, Allen E, Owens DK, Bravata DM. MetaAnalysis: Accuracy of Quantitative Ultrasound for Identifying Patients with Osteoporosis. Ann Intern Med. 2006;144:832-841

28. Gemalmaz A, Discigil G, Sensoy N, Basak O. Identifying osteoporosis in a primary care setting with quantitative ultrasound: relationship to anthropometric and lifestyle factors. J Bone Miner Metab. Apr 20. 2007;25(3):184-92. 29. Sinnott B, Kukreja S, Barengolts E. Utility of screening tools for the prediction of low bone mass in African American men. Osteoporos Int. 2006 Mar 8;17(5):684-92. 30. Pérez-Castrillón JL, Sagredo MG, Conde R, Del PinoMontes J, Luis D. OST Risk Index and Calcaneus Bone Densitometry in Osteoporosis Diagnosis. J Clin Densitom. 2007; 10(4):404-407.

31. Gudmundsdottir SL, Indridason OS, Franzson L, Sigurdsson G. Age-related decline in bone mass measured by dual-energy X-ray absorptiometry and quantitative ultrasound in a population-based sample of both sexes: identification of useful ultrasound thresholds for osteoporosis screening. J Clin Densitom. 2005; 8(1):80-86. 32. Shepherd AJ, Cass AR, Carlson CA, Ray L. Development and Internal Validation of the Male Osteoporosis Risk Estimation Score. Ann. Fam. Medicine. 2007; 5:540-546. 
33. Richy F, Gourlay M, Ross PD, Sen SS, Radican L, De Ceulaer F, Ben Sedrine W, Ethgen O, Bruyerel O, Reginster JY. Validation and comparative evaluation of the osteoporosis self-assessment tool (OST) in a Caucasian population from Belgium. Q J Med. 2004; 97:39-46.

34. Ghazi M, Mounach A, Nouijai A, Ghozlani I, Bennani L, Achemlal L, Bezza A, El Maghraoui A. Performance of the osteoporosis risk assessment tool in Moroccan men. Clin Rheumatol. 2007 Dec; 26: 2037-2041.

35. Skedros JG, Sybrowsky CL, Stoddard GJ. The osteoporosis self-assessment screening tool: a useful tool for the orthopaedic surgeon. J Bone Joint Surg Am. 2007 Apr; 89(4):765-72

36. Schousboe JT, Taylor BC, Fink HA, Kane RL, Cummings SR, Orwoll ES, Melton LJ 3rd, Bauer DC, Ensrud KE. Cost-effectiveness of bone densitometry followed by treatment of osteoporosis in older men. JAMA 2007 Aug 8;298(6):629-37.

37. Silva, Letícia K. Avaliação tecnológica em saúde: densitometria óssea e terapêuticas alternativas na osteoporose pós-menopausa. Cad. Saúde Pública. 2003 julago; 19(4):987-1003.

38. Tang BMP, Eslick GD, Nowson C, Smith C, Bensoussan A. Use of calcium or calcium in combination with vitamin $\mathrm{D}$ supplementation to prevent fractures and bone loss in people aged 50 anos and older: a meta-analysis. The Lancet. 2007 aug 25; 370(9588): 657-666.

\section{Endereço para Correspondência:}

Rua Marechal Deodoro, 144, apto. 202

Belo Horizonte - MG

CEP: 30150-110

\section{Endereço eletrônico:}

leosavassi@gmail.com 